\title{
An improved hybrid biogeography-based optimization algorithm for constrained optimization problems
}

\author{
Wen Long, Ximing Liang, Songjin Xu \\ Key Laboratory of Economics System Simulation, Guizhou University of Finance and Economics, \\ Guiyang Guizhou, 550004, China \\ School of Science, Beijing University of Architecture, Beijing, 100044, China \\ School of Mathematical Science, Tongren University, Tongren Guizhou, 554300, China
}

\begin{abstract}
Keywords: optimization algorithm, Constrained optimization problems
ABSTRACT: Constrained optimization problems are very important as they are encountered in many science and engineering applications. A hybrid method based on modified augmented Lagrangian multiplier and biogeography-based optimization (BBO) algorithm is proposed to solve constrained optimization problems. The basic steps of the proposed method are comprised of an outer iteration, in which the Lagrangian multipliers and various penalty parameters are updated using a first-order update scheme, and an inner iteration, in which a nonlinear optimization of the modified augmented Lagrangian function with simple bound constraints is implemented by BBO algorithm. Numerical results show that the proposed method is reliable and efficient for solving constrained optimization problems.
\end{abstract}

\section{INTRODUCTION}

Constrained optimization problems are always inevitable in many science and engineering disciplines, such as welded beam design problem, pressure vessel design problem, and so on. The general constrained optimization problem with equality, inequality, lower bound, and upper bound constraints is defined as

$$
\begin{array}{ll}
\min & f(\vec{x}) \\
\text { s.t. } & g_{j}(\vec{x})=0, j=1,2, \cdots, m_{e} \\
& h_{j}(\vec{x}) \geq 0, j=m_{e}+1, \cdots, m \\
& l_{i} \leq x_{i} \leq u_{i}, i=1,2, \cdots, n .
\end{array}
$$

where $\vec{x}=\left(x_{1}, x_{2}, \cdots, x_{n}\right)$ is a dimensional vector of $n$ decision variables, $f(\vec{x})$ is an objective function, $g_{j}(\vec{x}) \leq 0$ and $h_{j}(\vec{x})=0$ are known as inequality and equality constraints, respectively. $m_{e}$ is the number of equality constraints and $m-m_{e}$ is the number of inequality constraints, $l_{i}$ and $u_{i}$ are the lower bound and the upper bound of $x_{i}$, respectively.

Evolutionary algorithms have many advantages over conventional nonlinear programming techniques: easy implementation, reliable and robust performance, the gradients of the const function and constraint functions are not required, and the change of being trapped by a local minimum is lower. Due to those advantages, evolutionary algorithms have been successfully applied to solve constrained optimization problems in the past decade (Long et al. 2013; Daneshyari \& Yen 2012; Long et al. 2014). Biogeography-based optimization (BBO), proposed by Simon (Simon, 2008), is a new global optimization algorithm based on the biogeography theory, which is the study of the geographical distribution of biological organisms. BBO algorithm has shown good performance both on benchmark problems (Ma \& Simon 2011) and on real-world problems, including power system optimization (Roy et al. 2009) and mechanical gear train design (Savsani et al. 2009).

It is necessary to note that BBO algorithms are unconstrained search methods and lack an explicit mechanism to bias the search in constrained search space. The most common constraint-handling techniques are penalty-function-based methods because of their simplicity and ease of implementation. The augmented Lagrangian is an interesting penalty function that avoids the side-effects associated with ill-conditioning of simple penalty and barrier functions (Costa et al. 2012). In this paper, based on our previous work (Long et al. 2013), we integrate modified augmented Lagrangian multiplier method with BBO algorithm (denoted as MALBBO) for solving nonlinear constrained optimization problems. 


\section{THE PROPOSED MALBBO ALGORITHM}

\subsection{Modified augmented Lagrangian formulation}

In formula (1), if the simple bound (1d) is not present, then one can use the modified augmented Lagrange multiplier method to solve $(1 a)-(1 c)$. For given Lagrange multiplier vector $\lambda^{k}$ and penalty parameter vector $\sigma^{k}$, the unconstrained penalty sub-problem at the $k$-th step of this method is:

$$
\min P\left(x, \lambda^{k}, \sigma^{k}\right)
$$

where $P(x, \lambda, \sigma)$ is the following modified augmented Lagrangian function:

$$
P(x, \lambda, \sigma)=f(x)-\sum_{i=1}^{m_{e}}\left[\lambda_{i} c_{i}(x)-\frac{1}{2} \sigma_{i}\left(c_{i}(x)\right)^{2}\right]-\sum_{i=m_{e}+1}^{m} \tilde{P}_{i}(x, \lambda, \sigma)
$$

and $\tilde{P}_{i}(x, \lambda, \sigma)$ is defined as follows:

$$
\tilde{P}_{i}(x, \lambda, \sigma)=\left\{\begin{array}{lc}
\lambda_{i} c_{i}(x)-\frac{1}{2} \sigma_{i}\left(c_{i}(x)\right)^{2}, & \text { if } \lambda_{i}-\sigma_{i} c_{i}(x)>0 \\
\frac{1}{2} \lambda_{i}^{2} / \sigma_{i}, & \text { otherwise. }
\end{array}\right.
$$

If the simple bound $(1 d)$ is present, the above modified augmented Lagrange multiplier method needs to be modified. In modified barrier function methods, the simple bound constraints are treated as the general inequality constraints $x_{i}-l_{i} \geq 0$ and $u_{i}-x_{i} \geq 0$, which enlarges greatly the number of Lagrange multipliers and penalty parameters. So, we make another modification to deal with the bound constraints. At the $k$-th step, assume that the Lagrange multiplier vector $\lambda^{k}$ and penalty parameter vector $\sigma^{k}$ are given, we solve the following bound constrained sub-problem instead of (2):

$$
\begin{cases}\min & P\left(x, \lambda^{k}, \sigma^{k}\right) \\ \text { s.t. } & l_{i} \leq x_{i} \leq u_{i}\end{cases}
$$

where $P(x, \lambda, \sigma)$ is the same modified augmented Lagrangian function as in (3). The solution $x^{*}$ to sub-problem (5) can be obtained by searching the search space if $\lambda^{*}$ is known and $\sigma$ is large enough.

\subsection{Biogeography-based optimization (BBO) algorithm}

To obtain the global minimum for the $k$-th bound constrained minimization sub-problem (5), one can employ many solvers based on genetic algorithm, differential evolution algorithm or particle swarm optimization algorithm. Here we choose the biogeography-based optimization algorithm for the global search in (5). The objective function in (5), i.e., the modified augmented Lagrangian function $P\left(x, \lambda^{k}, \sigma^{k}\right)$ will be taken as the fitness evaluation, and the search space is defined by the lower and upper bounds of the variables $l_{i} \leq x_{i} \leq u_{i}$.

Biogeography-based optimization (BBO) algorithm (Simon, 2008) is a population-based stochastic optimization technique developed by Simon in 2008, inspired by the immigration and emigration of species between islands in search of more friendly habitats. Each solution is called a "habitat" with a habitat suitability index (HSI) and represented by an n-dimension real vector. The variables of the individual that characterize habitability are called suitability index variables $(S I V S)$. An initial individual of the habitat vectors is randomly generated. Those good solutions are considered to be habitats with a high HSI. Those poor ones are considered to be habitats with a low HSI. The solutions with high HSI tends to share their features with those with low HSI. In BBO, a habitat $H$ is a vector $(S I V S)$ which follows migration and mutation step to reach the optimal solution. The new candidate habitat is generation from all of the salutation in population by using the migration and mutation operators (Simon 2008). The flow chart of BBO algorithm is described in reference (Simon 2008).

\subsection{The framework of proposed MALBBO algorithm}

The proposed hybrid approach (called MALBBO) is performed in two stages. The outer stage is performed, which updates the Lagrange multipliers and penalty parameters, checks for convergence and reinitiates another bound constrained minimization accordingly or declares convergence. Following this, the inner stage is the bound constrained global minimization of the modified augmented Lagrangian function, in which a new iterative point near to the global minimum is found via biogeography-based optimization algorithm. The framework of the proposed MALBBO algorithm can be described in Figure 1. 


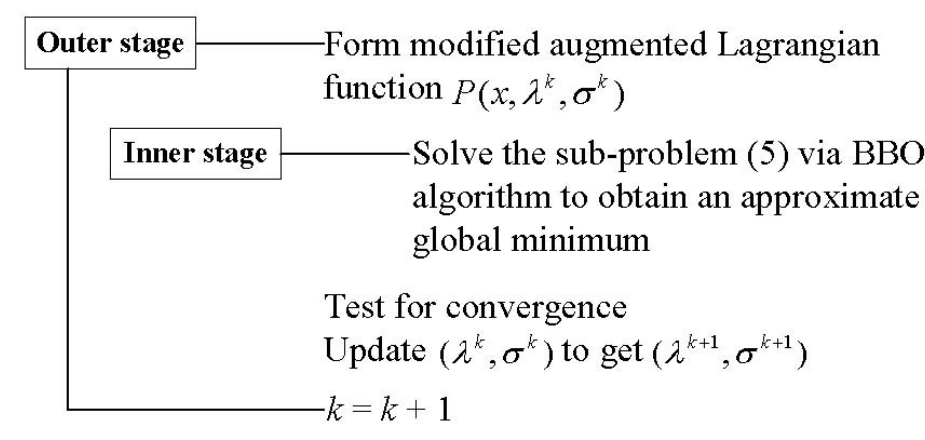

Figure 1. Framework of proposed MALBBO algorithm.

\section{SIMULATION EXPERIMENTS AND COMPARISONS}

To evaluate the performance of the proposed MALBBO algorithm, we use four well-known benchmark problems (denoted by g02, g03, g09 and g11) as extended by Runarsson and Yao (2000) for numerical simulation. The details expressions of four problems see (Runarsson \& Yao 2000).

Note that test problems g02 and g03 are maximization problems, and the others are minimization problems. In this study, the maximization problems are transformed into minimization using $-f(\vec{x})$. In addition, only test problems g03 and g06 contain equality constraints. For each test problem, 30 independent runs are performed in Matlab 7.0. The parameters used by MALBBO algorithm are the following: the population size was set to 100. The individuals are randomly initialized within the boundaries for each run according to a uniform probability distribution. The user-required tolerance $\varepsilon=1 e-8$, the initial Lagrange multiplier vector $\lambda^{0}=(1,1, \cdots, 1)$, the initial penalty parameter vector $\sigma^{0}=(10,10, \cdots, 10)$, the maximum allowed penalty parameter $\sigma_{u}=1 e 10$, the penalty parameter increasing factor $\gamma=10$, the reduction factor for feasibility norm $\zeta=0.25$, maximum immigration rate and maximum emigration rate of 1 , maximum number of fitness function evaluations, we use 120000 .

We compare our approach against seven state-of-the-art algorithms: stochastic ranking (SR) (Runarsson \& Yao 2000), blended BBO algorithm (B-BBO) (Ma \& Simon 2011), modified augmented Lagrangian with differential evolution (MALDE) (Long et al. 2013), hybrid genetic pattern search augmented Lagrangian (HGPSAL) (Costa et al. 2012), improved vector particle swarm optimization (IVPSO) (Sun et al. 2011), modified artificial bee colony (MABC) (Karaboga \& Akay 2011), and modified augmented Lagrangian with hybrid cuckoo search algorithm (MALHCS) (Long et al. 2014). Table 1 shows the results of the test problems in terms of the best, the mean, the worst, the standard deviation (st.dev) of the objective value of the solutions and the average number of function fitness evaluations (Avg.FFEs.). We report the solutions obtained by MALBBO, as well as those obtained by other seven approaches. 
Table 1. Comparing of the results of MALBBO with respect to seven other algorithms for four problems.

\begin{tabular}{|c|c|c|c|c|c|c|}
\hline $\begin{array}{c}\text { Problems/ } \\
\text { optimal }\end{array}$ & Algorithms & Best & Mean & Worst & St.dev & Avg.FFEs. \\
\hline \multirow{7}{*}{-0.803619} & $\begin{array}{c}\text { SR } \\
\text { B-BBO }\end{array}$ & $\begin{array}{l}-0.803515 \\
-0.803619\end{array}$ & $\begin{array}{l}-0.781975 \\
-0.802154\end{array}$ & $\begin{array}{l}-0.726288 \\
-0.801924\end{array}$ & $\begin{array}{l}2.0 \mathrm{E}-02 \\
\text { NA }\end{array}$ & $\begin{array}{c}350000 \\
50000\end{array}$ \\
\hline & MALDE & -0.803619 & -0.757552 & -0.659735 & $\begin{array}{l}3.743 \mathrm{E}- \\
02\end{array}$ & 120000 \\
\hline & HGPSAL & -0.611330 & -0.556323 & -0.526660 & $\begin{array}{c}2.501 \mathrm{E}- \\
02\end{array}$ & 227247 \\
\hline & IVPSO & -0.803619 & -0.769889 & -0.703477 & $\begin{array}{c}4.676 \mathrm{E}- \\
03\end{array}$ & 160000000 \\
\hline & MABC & -0.803611 & -0.795430 & -0.770319 & $\begin{array}{c}9.466 \mathrm{E}- \\
03\end{array}$ & 350000 \\
\hline & MALHCS & -0.803619 & -0.762088 & -0.640249 & 4.10E-02 & 150000 \\
\hline & MALBBO & -0.803619 & -0.798053 & -0.726029 & $\begin{array}{c}3.191 \mathrm{E}- \\
03\end{array}$ & 120000 \\
\hline \multirow[t]{2}{*}{$\begin{array}{c}\mathrm{g} 03 / \\
-1.0005001\end{array}$} & $\begin{array}{c}\text { SR } \\
\text { B-BBO } \\
\text { MALDE } \\
\text { HGPSAL } \\
\text { IVPSO } \\
\text { MABC } \\
\text { MALHCS }\end{array}$ & $\begin{array}{c}-1.000 \\
-1.0005 \\
-1.000000 \\
-1.000000 \\
-1.005010 \\
-1.000 \\
-1.000000\end{array}$ & $\begin{array}{c}-1.000 \\
-1.0004 \\
-1.000000 \\
-1.000000 \\
-1.005010 \\
-1.000 \\
-1.000000\end{array}$ & $\begin{array}{c}-1.000 \\
-1.0001 \\
-1.000000 \\
-1.000000 \\
-1.005010 \\
-1.000 \\
-1.000000\end{array}$ & $\begin{array}{c}1.9 \mathrm{E}-04 \\
\text { NA } \\
0.0 \mathrm{E}+00 \\
0.0 \mathrm{E}+00 \\
0.0 \mathrm{E}+00 \\
0.0 \mathrm{E}+00 \\
1.00 \mathrm{E}-10\end{array}$ & $\begin{array}{c}350000 \\
50000 \\
120000 \\
199439 \\
160000000 \\
350000 \\
150000\end{array}$ \\
\hline & MALBBO & -1.0005001 & -1.0005001 & -1.0005001 & $\begin{array}{c}1.874 \mathrm{E}- \\
12\end{array}$ & 120000 \\
\hline \multirow[b]{3}{*}{$\begin{array}{c}\mathrm{g} 09 / \\
680.630057\end{array}$} & $\begin{array}{c}\text { SR } \\
\text { B-BBO }\end{array}$ & $\begin{array}{l}680.630 \\
680.630\end{array}$ & & & $\begin{array}{c}\text { 3.4E-02 } \\
\text { NA }\end{array}$ & $\begin{array}{c}350000 \\
50000\end{array}$ \\
\hline & MALDE & 680.630057 & 680.630057 & 680.630057 & $\begin{array}{c}4.060 \mathrm{E}- \\
10\end{array}$ & 120000 \\
\hline & $\begin{array}{l}\text { HGPSAL } \\
\text { IVPSO } \\
\text { MABC } \\
\text { MALHCS } \\
\text { MALBBO }\end{array}$ & $\begin{array}{c}680.6301 \\
680.630058 \\
680.631 \\
680.6301 \\
680.630057\end{array}$ & $\begin{array}{c}680.6301 \\
680.630077 \\
680.636 \\
680.6301 \\
680.630057\end{array}$ & $\begin{array}{c}680.6301 \\
680.630139 \\
680.641 \\
680.6301 \\
680.630057\end{array}$ & $\begin{array}{l}0.0 \mathrm{E}+00 \\
3.0 \mathrm{E}-06 \\
2.6 \mathrm{E}-03 \\
1.43 \mathrm{E}-05 \\
9.9 \mathrm{E}-09\end{array}$ & $\begin{array}{c}56564 \\
160000000 \\
350000 \\
150000 \\
120000\end{array}$ \\
\hline \multirow[b]{3}{*}{$\underset{0.749900}{\mathrm{~g} 11 /}$} & $\begin{array}{c}\text { SR } \\
\text { B-BBO }\end{array}$ & $\begin{array}{c}0.750 \\
0.7499\end{array}$ & $\begin{array}{c}0.750 \\
0.7499\end{array}$ & $\begin{array}{c}0.750 \\
0.7499\end{array}$ & $\begin{array}{l}8.0 \mathrm{E}-05 \\
\text { NA }\end{array}$ & $\begin{array}{l}350000 \\
50000\end{array}$ \\
\hline & MALDE & 0.749900 & 0.749900 & 0.749900 & $\begin{array}{c}\text { 7.400E- } \\
09\end{array}$ & 120000 \\
\hline & $\begin{array}{l}\text { HGPSAL } \\
\text { IVPSO } \\
\text { MABC } \\
\text { MALHCS } \\
\text { MALBBO }\end{array}$ & $\begin{array}{c}0.750000 \\
0.749000 \\
0.750 \\
0.749999 \\
0.749900\end{array}$ & $\begin{array}{c}0.750000 \\
0.749000 \\
0.750 \\
0.750000 \\
0.749900\end{array}$ & $\begin{array}{c}0.750000 \\
0.749000 \\
0.750 \\
0.750000 \\
0.749900\end{array}$ & $\begin{array}{l}0.0 \mathrm{E}+00 \\
0.0 \mathrm{E}+00 \\
0.0 \mathrm{E}+00 \\
3.70 \mathrm{E}-09 \\
0.0 \mathrm{E}+00\end{array}$ & $\begin{array}{c}17948 \\
160000000 \\
350000 \\
150000 \\
120000\end{array}$ \\
\hline
\end{tabular}

*NA means Not Available.

As shown in Table 2, it can be observed that MALBBO consistently found the global optimal solutions in all problems. For the large feasible problem g02, methods SR, HGPSAL, and MABC are unable to reach the true optimum. With respect to SR, MALBBO provides better "best", "mean", "worst" and standard deviation value for all test problems. Compare with B-BBO, and HGPSAL, MAL-BBO finds better "mean" "worst", and "st.dev" results and similar "best" results for test functions g03, and g09. For test function g03, MALBBO provides better results than other seven approaches. The small values of standard deviations obtained by MAL-BBO in the majority of the problems highlight the consistency of the algorithm. As far as the computational effort (average number of function evaluations) is concerned, B-BBO seem to has the minimum computational effort, while IVPSO has considerable computational effort for all test functions (160000000). In general, the effort required by MALBBO is moderate among the constrained optimization approaches in comparison.

As a general remark on the comparison above, MALBBO shows a very competitive performance with respect to six state-of-the-art approaches in terms of the quality, the robustness, and the efficiency of search. 


\section{CONCLUSIONS}

This paper has presented a hybrid approach coupling modified augmented Lagrangian multiplier method and biogeography-based optimization algorithm for solving constrained optimization. The proposed MALBBO algorithm has demonstrated better performance than the other approaches in literature on solving four constrained optimization problems. In the future, we will apply MAL-BBO to various problems found in the real world. Meanwhile, we are interested in extending our method so that it can deal with multi-objective optimization problems.

\section{ACKNOWLEDGEMENT}

This work was financially supported by the National Natural Science Foundation of China (No. 61463009) and the Guizhou Provincial Fund of Science and Techonology(No. [2013]2082).

\section{REFERENCES}

Costa, L., Santo, L. \& Fernandes, E. 2012. A hybrid genetic algorithm pattern search augmented Lagrangian method for constrained global optimization. Applied Mathematics and Computation 218(8): 193-203.

Daneshyari, M. \& Yen G.G. 2012. Constrained multiple-swarm optimization within a cultural framework. IEEE Transactions on System, Man and Cybernetics. 42(2): 475-490.

Karaboga, D. \& Akay, B. 2011. A modified artificial bee colony (ABC) algorithm for constrained optimization problems. Applied Soft Computing 11(3): 3021-3031.

Long, W., Liang, X.M., Huang, Y.F. \& Chen, Y.X. 2013. A hybrid differential evolution augmented Lagrangian method for constrained numerical and engineering optimization. Computer-Aided Design. 45(12): 1562-1574.

Long, W., Liang, X.M., Huang, Y.F. \& Chen, Y.X. 2014. An effective hybrid cuckoo search algorithm for global constrained optimization. Neural Computing \& Applications 25(3-4): 911926.

Ma, H.P. \& Simon, D. 2011. Blended biogeography-based optimization for constrained optimization. Engineering Applications of Artificial Intelligence 24(3): 517-525.

Roy, P., Ghoshal, S. \& Thakur, S. 2009. Biogeography-based optimization for economic load dispatch problems. Electric Power Components and Systems 38(2): 166-181.

Runarsson, T.P. \& Yao, X. 2000. Stochastic ranking for constrained evolutionary optimization. IEEE Transactions on Evolutionary Computation 4(3): 284-294.

Savsani, V., Rao, R. \& Vakharia, D. 2009. Discrete optimization of a gear train using biogeographybased optimization technique. International Journal of Design Engineering 2(2): 205-223.

Simon, D. 2008. Biogeography-based optimization. IEEE Transactions on Evolutionary Computation 12(6): 702-713.

Sun, C.L., Zeng, J.C. \& Pan, J. 2011. An improved vector particle swarm optimization for constrained optimization problems. Information Sciences 181(6): 1153-1163. 